\section{THU0675 ASSOCIATION OF GLUCOSE HOMEOSTASIS MEASURES AND METABOLIC SYNDROME WITH KNEE CARTILAGE DEFECTS AND CARTILAGE VOLUME IN YOUNG ADULTS}

Tao Meng ${ }^{1}$, Alison Venn ${ }^{1}$, Flavia Cicuttini' ${ }^{2}$, Lyn March ${ }^{3}$, Marita Cross ${ }^{3}$, Terence Dwyer ${ }^{1,4}$, Graeme Jones ${ }^{1}$, Laura Laslett ${ }^{1}$, Benny Antony ${ }^{1}$, Changhai Ding ${ }^{1,5}$. ${ }^{1}$ Menzies Institute for Medical Research, University of Tasmania, Hobart, Australia; ${ }^{2}$ Department of Epidemiology and Preventive Medicine, Monash University, Melbourne, Australia; ${ }^{3}$ Institute of Bone and Joint Research, University of Sydney, Sydney, Australia; ${ }^{4}$ The George Institute for Global Health, Nuffield Department of Obstetrics and Gynaecology, University of Oxford, Oxford, United Kingdom; ${ }^{5}$ Clinical Research Centre, Zhujiang Hospital, Southern Medical University, Guangzhou, China

Background: Diabetes mellitus and knee osteoarthritis (OA) were commonly coexisting, and metabolic syndrome (MetS) shared many pathways with knee OA. However, the effects of glucose homeostasis and MetS on knee cartilage in young adults were unknown.

Objectives: To describe the associations of glucose homeostasis measures and MetS measures with knee cartilage defects and cartilage volume in young adults.

Methods: Australian young adults from the Childhood Determinants of Adult Health Study were selected to undergo knee magnetic resonance imaging (MRI) scans during 2008-2010 (aged 31-41 years). Fasting blood sample, waist circumference and blood pressure measures were collected during 2004-2006 (aged 26-36 years). Glucose, insulin, triglyceride and high-density lipoprotein cholesterol (HDL-C) were measured using serum samples. Homeostatic model assessment 2-insulin resistance (HOMA2-IR), HOMA2-beta cell function (HOMA2- $\beta$ ), HOMA2-insulin sensitivity (HOMA-S) were calculated using HOMA2 calculator (version 2.2.3 available from http://www.dtu.ox.ac.uk/homacalculator) according to fasting glucose and fasting insulin. MetS was defined when at least three of the following five components were present: high waist circumference (male $\geq 102 \mathrm{~cm}$, female $\geq 88 \mathrm{~cm})$, high fasting glucose $(\geq 5.6 \mathrm{mmol} / \mathrm{L})$, high serum triglycerides $(\geq 1.7 \mathrm{mmol} / \mathrm{L}$ ), low $\mathrm{HDL}-\mathrm{C}$ (male $<1.03 \mathrm{mmol} / \mathrm{L}$, female $<1.3$ $\mathrm{mmol} / \mathrm{L})$, and high blood pressure $(\geq 130 / 85 \mathrm{mmHg})$. Cartilage defects and cartilage volume were measured from MRI scans. Data were analysed using log binomial or linear regressions and were adjusted for age, gender, body mass index and physical activity.

Results: Among 328 participants (47.3\% were females), 40 (12.7\%) had hyperglycaemia and $21(6.7 \%)$ had MetS. Glucose homeostasis measures (except fasting glucose) were associated with tibiofemoral cartilage defects (Fasting insulin: relative risk (RR) $1.05 / \mathrm{mU} / \mathrm{L}, 95 \%$ confidence interval $(\mathrm{Cl})$ 1.01 to 1.08 ; HOMA2-IR: $1.44,1.08$ to 1.92 ; HOMA2- $\beta$ : $2.59,1.33$ to 5.07; HOMA2-S: $0.36,0.18$ to 0.72 ), but not patellar cartilage defects. There were no associations between glucose homeostasis measures and knee cartilage volume. MetS measures were not associated with either cartilage defects or cartilage volume, except the associations between high waist circumference and tibiofemoral cartilage defects (RR 2.32, 95\% $\mathrm{Cl} 1.18$ to 4.54 ) and between low HDL-C and tibiofemoral cartilage defects (RR $1.99,95 \% \mathrm{Cl} 1.08$ to 3.69 ).

Conclusion: Insulin resistance was associated with higher risk of tibiofemoral cartilage defects amongst young adults. MetS was not associated with neither cartilage defects nor cartilage volume. These suggest that glucose homeostasis, but not MetS, may play a role in cartilage damage in young adults and may lead to knee OA in later life.

Disclosure of Interests: None declared

DOI: 10.1136/annrheumdis-2019-eular.1679

\section{THU0676 \\ HEPATITIS B VIRUS REACTIVATION IN A COHORT OF PATIENTS TREATED WITH BIOLOGICS - DATA FROM THE ROMANIAN REGISTRY OF RHEUMATIC DISEASES}

Corina Mogosan ${ }^{1}$, Catalin Codreanu ${ }^{1}$, Denisa Predeteanu ${ }^{2}$, Simona Rednic ${ }^{3}$, Magda Parvu ${ }^{4}$, Elena Rezus ${ }^{5}$, Ruxandra lonescu ${ }^{2}$, Romanian Registry of Rheumatic Diseases. ${ }^{1}$ Clinical Center of Rheumatic Diseases "Dr. Ion Stoia", Rheumatology, Bucharest, Romania; 2"Sfanta Maria" Hospital, Rheumatology, Bucharest, Romania; ${ }^{3}$ County Emergency Hospital, Rheumatology, Cluj-Napoca, Romania; ${ }^{4}$ Clinical Hospital "Colentina", Rheumatology, Bucharest, Romania; ${ }^{5}$ Clinical Rehabilitation Hospital, Rheumatology, lasi, Romania

Background: Accompanying the increased use of biological and non-biological antirheumatic drugs, a greater number of cases of hepatitis B virus (HBV) reactivation have been reported in inactive hepatitis $B$ surface antigen ( $\mathrm{HBsAg}$ ) carriers and also in $\mathrm{HBsAg}$-negative patients (pts) who have resolved HBV infection (1). Romania has a high prevalence for HBV infection. According to a national epidemiological study conducted in
2013, 27,9\% from the Romanian population has serological markers of resolved HBV infection, while $4.2 \%$ of inactive carriers of $\mathrm{HBsAg}$ (2).

Objectives: To estimate the rate of HBV reactivation in a cohort of patients treated with biologics, in Romania.

Methods: Data were gathered from the Romanian Registry of Rheumatic Diseases (RRBR) for rheumatoid arthritis (RA), ankylosing spondylitis (AS) and psoriatic arthritis (PsA). The cohort included patients previously exposed to HBV: HBsAg inactive carriers or resolved HBV infection. HBV reactivation was considered as the presence of DNA-HBV or positivity of HBsAg in a previously negative patient. The collected data included exposure to biologics in person-years (PY) and serological markers of HBV. Results: The cohort included 1744pts (5505.95 PY): 936 RA pts (2762.97 PY), 640 AS pts (2037.64 PY) and 168 PsA pts (705.34 PY). The mean age was 58.72yrs (65yrs for RA, 49.58yrs for AS, 61.58yrs for PsA); $1058(60.6 \%)$ women: $786(74.2 \%)$ RA pts, $175(16.5 \%)$ AS pts and 97 (9.1\%) PsA pts. Mean disease duration was 14.5yrs for RA, 10.7yrs for AS and 10.5yrs for PsA.The prevalence of HBsAg inactive carriers were $44(4.7 \%)$ in RA group, $53(8.28 \%)$ in AS group and $6(3.57 \%)$ in PsA. The frequency of resolved HBV infection was $892(95.3 \%)$ in RA group, $587(91.7 \%)$ in AS and $162(96.4 \%)$ in PsA. The total number of observed HBV reactivation cases was $16(0.9 \%)$. In RA, $9(0.9 \%)$ cases of HBV reactivation (0.31/100 PY) were observed, all cases on resolved HBV infection state; 7 pts were treated with rituximab (RTX), 2 pts with TNFa blockers. 8 cases $(0.28 / 100 \mathrm{PY})$ of HBV reactivation were observed in those without antiviral prophylaxis, compared to a single reactivation case $(0.03 / 100 P Y)$ when antiviral prophylaxis was used. In AS cohort occurred $6(0.9 \%)$ cases of HBV reactivation (0.29/100 PY), all patients being treated with TNFa blockers ( 3 etanercept, 1 adalimumab, 1 infliximab, 1 golimumab): 4 cases of HBV reactivation developed in inactive carriers of $\mathrm{HBsAg}$ and 2 in resolved HBV infection. 5 cases (0.24/100PY) of HBV reactivation were observed in those without antiviral prophylaxis, compared to a single reactivation case $(0.04 / 100 \mathrm{PY})$ when antiviral prophylaxis was used. In PsA group, only 1 case of HBV reactivation (0.14/ $100 \mathrm{PY}$ ), on inactive carrier of $\mathrm{HBsAg}$ state, during TNFa blocking agent (golimumab).

Conclusion: HBV reactivation appeared more often on a resolved infection state, especially without antiviral prophylaxis. No cases of fulminan hepatitis were noted. Most cases developed in RTX exposed RA patients, while in AS group all HBV reactivation appeared with TNFa blockers exposure.

\section{REFERENCES:}

[1] Shunsuke M, Shigetoshi F. World J Gastroenterol 2015 September 28; 21 (36): 10274-10289

[2] https://www.cnscbt.ro/index.php

Disclosure of Interests: None declared

DOI: 10.1136/annrheumdis-2019-eular.5037

\begin{tabular}{|l|l}
\hline THU0677 & IDENTIFICATION OF PREVALENCE, PROGNOSTIC \\
& FACTORS, AND OUTCOMES OF PATIENTS WITH \\
& INTERSTITIAL PNEUMONIA WITH AUTOIMMUNE \\
& FEATURES: A SINGLE CENTER LARGE-SCALE \\
OBSERVATIONAL COHORT STUDY
\end{tabular}

Okinori Murata ${ }^{1,2}$, Katsuya Suzuki $^{1}$, Nobuhito Sasaki ${ }^{2}$, Makoto Maemondo ${ }^{2} .{ }^{1}$ Keio University School of Medicine, Division of Rheumatology, Department of Internal Medicine, Tokyo, Japan; ${ }^{2}$ wwate Medical University School of Medicine, Division of Pulmonary medicine, Allergy, and Rheumatology, Department of Internal Medicine, Morioka, Japan

Background: Patients with idiopathic interstitial pneumonia (IIP) may have features of connective tissue diseases (CTDs). The term interstitial pneumonia with autoimmune features (IPAF) has been recently proposed for such patients. A few studies have been reported in prevalence of IPAF which was varied from $7.3 \%$ to $34.1 \%$ [1, 2]. Factors reported to indicate a poor prognosis in IPAF include age, smoking history, organizing pneumonia pattern in HRCT, anti-RNP antibody positivity, decline in\%DLCO and presence of a multi-compartment feature within the morphological domain [2, 3]. To date, however, no study has comprehensively described prevalence of IPAF and factors of exacerbation.

Objectives: The aim of study was to identify of prevalence of IPAF in patients with interstitial lung disease, prognostic factors for exacerbation in patients with IPAF, and compared outcomes among patients with IPAF, IIP, and CTD-ILD.

Methods: Six hundreds- and seventy-two patients who visited our department between April 2009 and March 2018 and were evaluated by chest 